Ciência Florestal, Santa Maria, v. 26, n. 1, p. 69-80, jan.-mar., 2016

ISSN 0103-9954

\title{
PRODUÇÃO DE MUDAS DE UMBUZEIRO (Spondias tuberosa Arruda Câmara) EM DIFERENTES SUBSTRATOS E TAMANHO DE RECIPIENTES
}

\author{
SEEDLINGS PRODUCTION OF UMBUZEIRO (Spondias tuberosa Arruda Câmara) IN DIFFERENT \\ SUBSTRATES AND SIZE OF BAGS
}

\author{
Flávio Ricardo da Silva Cruz ${ }^{1}$ Leonaldo Alves de Andrade ${ }^{2}$ Ramon Costa Feitosa ${ }^{3}$
}

\begin{abstract}
RESUMO
A espécie Spondias tuberosa Arr. Cam. (umbuzeiro) destaca-se como uma das espécies arbóreas de maior potencial do semiárido brasileiro. Apesar de sua relevante importância socioeconômica e ambiental, há falta de estudos voltados para o estabelecimento de um modelo de produção de mudas da espécie. O objetivo desse trabalho foi avaliar a produção de mudas de Spondias tuberosa utilizando diferentes substratos e tamanho de recipientes. O experimento foi conduzido no viveiro do Laboratório de Ecologia Vegetal (LEV) do Centro de Ciências Agrárias (CCA) da Universidade Federal da Paraíba (UFPB), Areia - PB. O delineamento experimental usado foi em blocos casualizados em esquema fatorial $2 \times 7$ e parcelas subdivididas, sendo que os recipientes representaram as parcelas e os substratos as subparcelas. Foram utilizados substratos formulados a partir da mistura de terra de subsolo (37,5-100\%), areia lavada (12,5$25 \%$ ) e esterco bovino curtido (10-50\%). Os recipientes utilizados foram sacos de polietileno preto com volumes de $1900 \mathrm{~cm}^{3}(15 \times 27 \mathrm{~cm})$ e $5000 \mathrm{~cm}^{3}(25 \times 26 \mathrm{~cm})$. Para a obtenção das plântulas, sementes de Spondias tuberosa foram semeadas em sementeira de alvenaria até a repicagem (90 dias após o semeio). Aos 78 dias, após a repicagem, as mudas foram avaliadas quanto à altura, diâmetro do colo, relação altura/ diâmetro do colo, diâmetro do xilopódio, comprimento de raiz, massa seca da parte aérea e raiz. Os dados obtidos foram submetidos à análise de variância e ao teste $\mathrm{F}$, sendo as médias comparadas pelo teste de Tukey ao nível de $5 \%$ de probabilidade, utilizando o software SISVAR $^{\circledR}$. Os substratos com esterco bovino curtido proporcionaram os maiores valores em altura e comprimento de raiz. Para a produção de mudas de Spondias tuberosa, é recomendado o substrato contendo terra de subsolo (45\%) + Areia (15\%) + Esterco bovino (40\%).
\end{abstract}

Palavras-chave: Caatinga; espécies florestais; sacos de polietileno.

\begin{abstract}
The species Spondias tuberosa Arr. Cam. (umbuzeiro) stands out as one of the tree species with the greatest potential of the Brazilian semiarid. Even though it's significant socioeconomic and environmental importance, there is a lack of studies aimed at establishing a seedlings production model of this kind of plant. The purpose of this study was to evaluate the production of seedlings Spondias tuberosa using different substrates and size of bags. The experiment was conducted in the nursery of the Plant Ecology Laboratory (LEV) from the Agrarian Sciences Center (CCA) of the Federal University of Paraiba (UFPB), Areia/ PB. The experimental design was a randomized block in factorial $2 \times 7$ and subdivided blocks containers

1 Engenheiro Agrônomo, Mestre em Agronomia, Programa de Pós-Graduação em Agronomia, Universidade Federal da Paraíba - Campus II, Rodovia PB 079, Km 12, Caixa Postal 66, CEP 58397-000, Areia (PB), Brasil. flricardocruz@hotmail.com

2 Engenheiro Agrônomo, Dr. Professor Associado do Departamento de Fitotecnia e Ciências Ambientais, Universidade Federal da Paraíba - Campus II, Rodovia PB 079, Km 12, CEP 58397-000, Areia (PB), Brasil. Bolsista de Produtividade em Pesquisa do CNPq. landrade@cca.ufpb.br

3 Engenheiro Agrônomo, Doutorando pelo Programa de Pós-Graduação em Engenharia Agrícola, Universidade Federal do Ceará, Campus do Pici, Bloco 804, Caixa Postal 12.168, CEP 60450-760, Fortaliza (CE), Brasil. ramon.costa@hotmail.com
\end{abstract}

Recebido para publicação em 24/01/2013 e aceito em 17/12/2013

Ci. Fl., v. 26, n. 1, jan.-mar., 2016 
plots and subplots substrates. It were used substrates formulated from the starting mixture of subsoil land (37.5-100\%), sand (12.5-25\%) and tanned cattle manure (10-50\%). The containers used were black polyethylene bags with a volume of $1900 \mathrm{~cm}^{3}(15 \times 27 \mathrm{~cm})$ and $5000 \mathrm{~cm}^{3}(25 \times 26 \mathrm{~cm})$. To obtain seedlings of Spondias tuberosa seeds were sown in seed masonry until the transplant (90 days after sowing). At 78 days after transplanting the seedlings were evaluated for height, root collar diameter, relationship shoot/root collar diameter, xylopodium diameter, root length, dry weigh of shoot and root. The datas were subjected to analysis of variance and $\mathrm{F}$ test, measured and compared by Tukey test at $5 \%$ probability, using the software SISVAR $^{\circledR}$. The substrates with cattle manure provided higher values for height and root length. For the seedlings production of Spondias tuberosa, is recommended substrate subsoil land $(45 \%)+$ sand $(15 \%)+$ cattle manure (40\%).

Keywords: Caatinga; forest species; polyethylene bags.

\section{INTRODUÇÃO}

Dentre as espécies vegetais da caatinga o umbuzeiro (Spondias tuberosa Arruda Câmara) destaca-se pela importância, quer seja pelos frutos que produz, quer seja pela sua função ambiental e sociocultural que representa para as populações locais. O referido táxon é uma frutífera pertencente à família Anacardiaceae adaptada a períodos prolongados de seca devido aos xilopódios presentes no seu sistema radicular que possuem alta capacidade de armazenamento de água e à caducifólia acentuada (LORENZI, 1998; PAULA et al., 2007). O umbuzeiro apresenta-se como uma espécie de grande potencial destacandose pela possibilidade de ser cultivado em larga escala, podendo ser aproveitado tanto para alimentação humana quanto para a suplementação alimentar de caprinos e ovinos, que constituem os rebanhos predominantes do semiárido brasileiro (CAVALCANTI et al., 2002b). Os frutos da espécie são comercializados por pequenos agricultores nas principais cidades do Nordeste do Brasil, podendo ser consumidos in natura ou servir de base para a elaboração de sucos, sorvetes, geleias, doces, compotas e a tradicional umbuzada (CAVALCANTI et al., 2000; FOLEGATTI et al., 2003).

Ao longo do tempo, os ecossistemas brasileiros vêm sofrendo sérios danos decorrentes das ações antrópicas, sendo que, dentre eles, o bioma caatinga é um dos mais ameaçados devido, sobretudo, ao uso mal planejado de seus recursos, principalmente no que tange à remoção da vegetação autóctone (SANTANA e SOUTO, 2006; XAVIER et al., 2011). Nesse contexto, o umbuzeiro apresenta-se como uma das espécies da caatinga cuja população está bastante reduzida, sendo necessários programas de incentivo à preservação do referido táxon (MELO et al., 2005).

Apesar do notável potencial econômico e ambiental do umbuzeiro há carência de estudos voltados para o estabelecimento de protocolos de produção de mudas que possam servir para o estabelecimento de pomares comerciais e recuperação de áreas desmatadas da caatinga. Sabe-se da importância de insumos de qualidade em toda a cadeia produtiva, tanto na agropecuária, quanto no setor florestal e, quando se trata de mudas, isto não constitui exceção. Mudas de qualidade são essenciais para assegurar o sucesso das ações de revegetação, resgate de germosplasma e estabelecimento de povoamentos, quer sejam com fins comerciais ou conservacionistas. Dessa forma, a definição de estratégias que favoreçam a produção de mudas com qualidade em intervalos de tempo reduzidos é de relevante importância (CUNHA et al., 2005).

Para a produção de mudas, o tipo de substrato e o tamanho do recipiente influenciam diretamente na qualidade e no custo final desse insumo. $O$ substrato interfere no crescimento de mudas devido a características como estrutura, textura, capacidade de retenção de água e grau de infestação de patógenos sendo, portanto, um dos fatores mais complexos na produção de mudas (ALBUQUERQUE et al., 1998; CARVALHO FILHO et al., 2003; RODRIGUES et al., 2007). Cada espécie apresenta um comportamento diferente sobre um mesmo substrato, sendo necessário verificar cientificamente qual substrato ou combinação destes possibilita a obtenção de mudas com maior qualidade (SMIDERLE et al., 2001; CUNHA et al., 2006). Muitas vezes, no processo de produção de mudas, faz-se uso de materiais orgânicos objetivando a obtenção de maiores incrementos na produção. A adição de fontes de matéria orgânica na composição 
de substratos, a exemplo do esterco bovino curtido, é uma das práticas que contribui no fornecimento de nutrientes às plantas e melhoria das características físicas do meio de cultivo (CAVALCANTI et al., 2002a; LIMA et al., 2006a).

Quanto aos recipientes, Pereira et al. (2010) relatam que a sua escolha traz implicações de ordem técnica e econômica, sendo ideais os que harmonizam o custo de produção. Os sacos de polietileno são os recipientes mais utilizados para a produção de mudas, em pequena ou média escala, não obstante, esse tipo de recipiente possa influenciar na qualidade das mudas produzidas, culminando em uma maior ou menor taxa de sobrevivência e crescimento inicial diferenciado, após o plantio (JOSÉ et al., 2005). Para cada espécie, o volume do recipiente pode influenciar na percentagem de sobrevivência das mudas no campo sendo, portanto, necessários estudos específicos tendo em vista determinar o volume do recipiente mais adequado para o crescimento das diferentes espécies vegetais (NICOLOSO et al., 2000; LIMA et al., 2006b).

Diante do exposto o objetivo desse trabalho foi avaliar o crescimento de mudas de Spondias tuberosa, utilizando sacos de polietileno de diferentes volumes e diferentes composições de substratos.

\section{MATERIAL E MÉTODOS}

O experimento foi conduzido no viveiro florestal do Laboratório de Ecologia Vegetal (LEV), do Departamento de Fitotecnia e Ciências Ambientais (DFCA), do Centro de Ciências Agrárias (CCA) da Universidade Federal da Paraíba
(UFPB), Areia - PB, em ambiente de pleno sol de outubro de 2009 a janeiro de 2010. O clima local, pela classificação de Köppen, é do tipo AS', isto é, clima tropical, semiúmido, com estação chuvosa no período outono-inverno. Durante o período de condução da pesquisa, foram coletados, na estação meteorológica do referido Centro, dados referentes à temperatura média, expressa em graus Celsius, umidade relativa do ar e índices pluviométricos (Figura 1).

Os frutos de umbuzeiro foram provenientes de dois municípios paraibanos. Um lote foi coletado na zona rural do município de Pocinhos - PB (microrregião do Curimataú Ocidental) diretamente de árvores-matrizes e sob a área de projeção da copa. O segundo lote foi coletado na cidade de São Sebastião do Umbuzeiro - PB (microrregião do Cariri Ocidental) e adquirido em feira livre na cidade de Areia -PB. Os frutos foram levados para o Laboratório de Ecologia Vegetal, no qual foram manualmente beneficiados sendo que, após esta etapa, utilizando água e peneira, as sementes foram completamente limpas e colocadas para secar a sombra por dois dias.

Para a obtenção das plântulas de Spondias tuberosa, logo depois da secagem, as sementes foram embebidas em água por 48 horas (COSTA et al., 2001) e, em seguida, semeadas a lanço em sementeiras de alvenaria tendo como substrato areia lavada. Após a disposição das sementes na sementeira, as mesmas foram cobertas com uma camada de areia de modo que não ficassem visíveis. A quantidade de plântulas necessárias para o experimento foi obtida 90 dias após o semeio. Após a emergência, surgimento do primeiro par

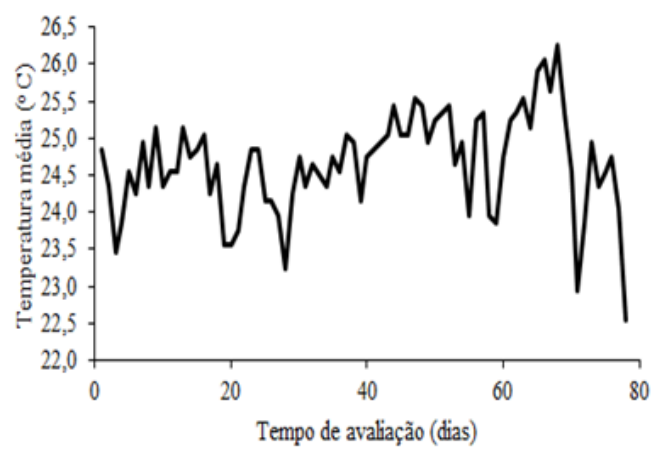

B

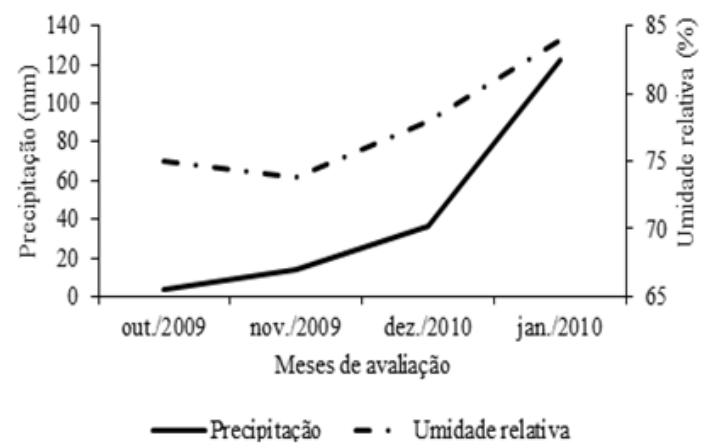

FIGURA 1: Dados meteorológicos referentes à temperatura média (A), precipitação e umidade relativa do ar (B) durante o período de condução do experimento.

FIGURE 1: Meteorological data regarding average temperature (A), precipitation and air relative humidity (B) during the period of the experiment conduction. 
de folhas e quando as plântulas apresentaram uma altura de aproximadamente $5 \mathrm{~cm}$, as mesmas foram repicadas para sacos de polietileno contendo diferentes composições de substratos. Na ocasião da repicagem, as plântulas de Spondias tuberosa foram cobertas com tela agrícola (sombrite - 50\%) por duas semanas, objetivando diminuir o estresse causado pela incidência direta de raios solares e favorecer o índice de pega.

Foram utilizadas sete composições de substratos, a seguir relacionados: $\mathrm{S}_{1}$ - Terra de subsolo (100\%); $\mathrm{S}_{2}$ - Terra de subsolo (75\%) + Areia lavada (25\%); $\mathrm{S}_{3}$ - Terra de subsolo $(75 \%)+$ Areia lavada $(15 \%)+$ Esterco bovino $(10 \%) ; \mathrm{S}_{4}-$ Terra de subsolo $(60 \%)+$ Areia lavada $(20 \%)+$ Esterco bovino (20\%); $\mathrm{S}_{5}$ - Terra de subsolo $(52,5 \%)+$ Areia lavada $(17,5 \%)+$ Esterco bovino $(30 \%) ; \mathrm{S}_{6}$ - Terra de subsolo $(45 \%)+$ Areia lavada $(15 \%)+$ Esterco bovino (40\%); $\mathrm{S}_{7}$ - Terra de subsolo $(37,5 \%)+$ Areia lavada (12,5\%) + Esterco bovino (50\%). Os substratos foram submetidos à análise de fertilidade no Laboratório de Química e Fertilidade do Solo do Centro de Ciências Agrárias da Universidade Federal da Paraíba (Tabela 2). Levando em consideração a possibilidade de um rápido crescimento das mudas de Spondias tuberosa em função da utilização do esterco bovino na composição dos substratos, foram utilizados sacos de polietileno (preto) com volumes de $1900 \mathrm{~cm}^{3}(15 \times 27 \mathrm{~cm})$ e $5000 \mathrm{~cm}^{3}(25 \times 26 \mathrm{~cm})$.

Aos 78 dias após a repicagem, as plantas de Spondias tuberosa foram avaliadas quanto à altura $(\mathrm{H})$ - medida com uma régua graduada em centímetros a partir do colo da planta até o meristema apical (cm planta-1); comprimento da raiz (CR) aferido com uma régua graduada em centímetros a partir do colo até a extremidade da raiz mais longa $\left(\mathrm{cm}\right.$ planta ${ }^{-1}$ ); diâmetro do colo (DC) - medido na base do caule da planta e determinado com o auxílio de um paquímetro digital ( $\left.\mathrm{mm} \mathrm{planta}^{-1}\right)$; e diâmetro do xilopódio (DX) - medido com o auxílio de paquímetro digital sendo considerado o maior diâmetro $\left(\mathrm{cm}\right.$ planta $\left.^{-1}\right)$. Após este procedimento, a parte aérea e as raízes foram previamente lavadas (objetivando remover o substrato aderido às mesmas), postas separadamente em sacos de papel Kraft e levadas para estufa de circulação forçada de ar regulada a uma temperatura de $65^{\circ} \mathrm{C}$ (até atingir massa seca constante) para a obtenção da massa seca da parte aérea (MSPA) e massa seca de raízes (MSRA), expressos em g planta ${ }^{-1}$. Além dessas características foi determinada a relação altura/ diâmetro do colo (H/DC).
O delineamento experimental foi em blocos casualizados em esquema fatorial $2 \times 7$ e parcelas subdivididas, em que as parcelas principais foram os recipientes e os substratos as subparcelas. A unidade experimental foi constituída por seis mudas. Os dados obtidos foram submetidos à análise de variância e ao teste $\mathrm{F}(\mathrm{p} \leq 0,05)$ sendo as médias comparadas pelo teste de Tukey ao nível de 5\% de probabilidade, utilizando o software SISVAR $^{\circledR}$ (FERREIRA, 2007).

\section{RESULTADOS E DISCUSSÃO}

A Tabela 1 mostra o resumo da análise de variância para as variáveis avaliadas no experimento. Em relação à altura $(\mathrm{H})$ de plantas de Spondias tuberosa, houve efeito significativo ao nível $1 \%$ de probabilidade, para a interação recipiente $\mathrm{x}$ substrato.

Os substratos com esterco em sua composição apresentaram os maiores teores de nutrientes em relação aos que não o tinham. $\mathrm{Na}$ Tabela 2 estão os dados da análise química dos substratos utilizados.

$\mathrm{O}$ teste de média realizado com os valores de altura obtidos 78 dias após a repicagem mostra que as plantas que se desenvolveram no substrato $\mathrm{S}_{6}-$ Terra $(45 \%)+$ Areia $(15 \%)+$ Esterco bovino $(40 \%)$ e no recipiente com volume de $5000 \mathrm{~cm}^{3}$ apresentaram maior valor médio de altura o qual correspondeu a 44,52 cm (Tabela 3). Este valor não diferiu estatisticamente dos obtidos em plantas de Spondias tuberosa que se desenvolveram nos substratos $\mathrm{S}_{4}$ - Terra $(70 \%)+$ Areia lavada $(20 \%)+$ Esterco bovino (20\%) e $\mathrm{S}_{7}$ - Terra $(37,5 \%)+$ Areia lavada $(12,5 \%)+$ Esterco bovino $(50 \%)$.

Os substratos contendo esterco bovino curtido proporcionaram um incremento de até 19,37 cm na altura de plantas de Spondias tuberosa quando comparados com aqueles que não tinham esterco em sua composição, o que está relacionado com a maior presença de nutrientes no esterco bovino, conforme a análise dos substratos. Além disso, esses substratos apresentaram valores de saturação por base acima de 60\% (Tabela 2). Ferraz et al. (2005) destacaram que substratos com altos valores de nutrientes e saturação por bases tendem a propiciar crescimento satisfatório das plantas.

Quanto aos volumes dos recipientes avaliados e independentemente dos substratos, plantas de Spondias tuberosa que se desenvolveram nos recipientes com $5000 \mathrm{~cm}^{3}$ tiveram um 
TABELA 1: Quadrados médios $(\mathrm{QM})$ e coeficientes de variação $(\mathrm{CV})$ referentes à altura de plantas $(\mathrm{H})$, diâmetro do colo (DC), comprimento de raiz (CR), diâmetro de xilopódio (DX), massa seca da parte aérea (MSPA), massa seca de raízes (MSRA) e relação altura/diâmetro do colo (H/ DC) de mudas de Spondias tuberosa em dois volumes de recipientes e sete composições de substratos, 78 dias após a repicagem.

TABLE 1: Mean squares (QM) and coefficients of variation (CV) referring to the height of plants (H), collar diameter (DC), root length (CR), xylopodium diameter (DX), dry mass of shoot (MSPA), and dry mass of roots (MSR) and relation height/collar diameter (H/DC) seedlings Spondias tuberosa in two volumes of containers and seven substrate compositions, 78 days after the transplant.

\begin{tabular}{lcccccccc}
\hline \multirow{2}{*}{ FV } & GL & \multicolumn{7}{c}{ QM } \\
\cline { 3 - 9 } & & $\mathrm{H}$ & $\mathrm{DC}$ & $\mathrm{CR}$ & $\mathrm{DX}$ & MSPA & MSR & H/DC \\
\hline Bloco & 3 & 19,916 & 0,637 & 75,406 & 0,056 & 0,939 & 4,195 & 0,900 \\
VR. & 1 & $469,800^{* *}$ & $11,657^{* *}$ & $3,615^{\text {ns }}$ & $0,401^{*}$ & $99,191^{* *}$ & $4,582^{\text {ns }}$ & $0,288^{\text {ns }}$ \\
Erro 1 & 3 & 6,336 & 0,262 & 18,031 & 0,029 & 1,588 & 1,298 & 0,370 \\
S. & 6 & $783,389^{* *}$ & $20,782^{* *}$ & $56,876^{* *}$ & $1,668^{* *}$ & $94,193^{* *}$ & $32,110^{* *}$ & $2,152^{* *}$ \\
VR. x S. & 6 & $18,328^{*}$ & $1,518^{\text {ns }}$ & $13,853^{\text {ns }}$ & $0,220^{* *}$ & $7,701^{* *}$ & $2,705^{*}$ & $0,390^{\text {ns }}$ \\
Erro 2 & 36 & 6,142 & 0,918 & 16,362 & 0,046 & 1,015 & 1,007 & 0,5965 \\
CV1\% & - & 8,25 & 7,66 & 14,43 & 6,48 & 24,66 & 27,34 & 13,56 \\
CV2\% & - & 8,12 & 14,33 & 13,75 & 8,12 & 19,72 & 24,08 & 17,20 \\
\hline
\end{tabular}

Em que: ${ }^{\text {ns }}$ valor de $\mathrm{F}$ não significativo a 5 e $1 \%$ de probabilidade; *valor de $\mathrm{F}$ significativo a $5 \%$ de probabilidade;**valor de F significativo a $1 \%$ de probabilidade; $\mathrm{FV}$ = fonte de variação; $\mathrm{GL}=$ grau de liberdade; $\mathrm{VR}$ = volume do recipiente; $\mathrm{S}=$ substrato.

TABELA 2: Caracterização química dos substratos utilizados na produção de mudas de Spondias tuberosa. TABLE 2: Chemical characterization of substrates used in the seedlings production of Spondias tuberosa.

\begin{tabular}{|c|c|c|c|c|c|c|c|}
\hline \multirow{2}{*}{ Características } & \multicolumn{7}{|c|}{ Substrato } \\
\hline & $\mathrm{S}_{1}$ & $\mathrm{~S}_{2}$ & $\mathrm{~S}_{3}$ & $\mathrm{~S}_{4}$ & $\mathrm{~S}_{5}$ & $\mathrm{~S}_{6}$ & $\mathrm{~S}_{7}$ \\
\hline $\mathrm{pH}-\mathrm{H}_{2} \mathrm{O}_{(1: 2,5)}$ & 4,57 & 4,43 & 4,87 & 4,67 & 4,84 & 4,66 & 6,84 \\
\hline $\mathrm{P}\left(\mathrm{mg} \mathrm{dm}^{-3}\right)$ & 12,43 & 6,20 & 61,08 & 121,00 & 36,24 & 287,20 & 522,27 \\
\hline $\mathrm{K}^{+}\left(\mathrm{mg} \mathrm{dm}^{-3}\right)$ & 144,16 & 35,81 & 383,00 & 1274,00 & 1021,00 & 255,45 & 2248,00 \\
\hline $\mathrm{Na}^{+}\left(\mathrm{cmol}_{\mathrm{c}} \mathrm{dm}^{-3}\right)$ & 0,29 & 0,17 & 0,28 & 0,66 & 0,80 & 1,23 & 1,67 \\
\hline $\mathrm{H}^{+}+\mathrm{Al}^{+3}\left(\mathrm{cmol}_{\mathrm{c}} \mathrm{dm}^{-3}\right)$ & 7,43 & 3,71 & 2,81 & 3,22 & 2,64 & 3,55 & 0,99 \\
\hline $\mathrm{Al}^{+3}\left(\mathrm{cmol}_{\mathrm{c}} \mathrm{dm}^{-3}\right)$ & 0,60 & 0,60 & 0,10 & 0,05 & 0,00 & 0,05 & 0,00 \\
\hline $\mathrm{Ca}^{+2}\left(\mathrm{cmol}_{\mathrm{c}} \mathrm{dm}^{-3}\right)$ & 1,20 & 0,90 & 1,80 & 1,60 & 4,15 & 5,90 & 5,20 \\
\hline $\mathrm{Mg}^{+2}\left(\mathrm{cmol}_{\mathrm{c}} \mathrm{dm}^{-3}\right)$ & 0,85 & 0,80 & 1,65 & 5,55 & 5,15 & 7,50 & 8,35 \\
\hline $\mathrm{SB}\left(\mathrm{cmol}_{\mathrm{c}} \mathrm{dm}^{-3}\right)$ & 2,71 & 1,96 & 4,71 & 11,07 & 12,71 & 15,28 & 20,97 \\
\hline CTC $\left(\mathrm{cmol}_{\mathrm{c}} \mathrm{dm}^{-3}\right)$ & 10,14 & 5,67 & 7,52 & 4,29 & 15,35 & 18,83 & 21,96 \\
\hline $\mathrm{V}(\%)$ & 26,73 & 34,57 & 62,63 & 77,47 & 82,80 & 81,15 & 95,49 \\
\hline m (\%) & 18,13 & 23,44 & 2,08 & 0,45 & 0,00 & 0,33 & 0,00 \\
\hline M.O. $\left(\mathrm{g} \mathrm{kg}^{-1}\right)$ & 27,35 & 14,47 & 1,71 & 24,61 & 24,29 & 45,00 & 86,46 \\
\hline
\end{tabular}

Em que: Análise realizada no Laboratório de Química e Fertilidade do Solo do Centro de Ciências Agrárias da Universidade Federal da Paraíba. Em que: $\mathrm{S}_{1}$ - TS (100\%); $\mathrm{S}_{2}$ - TS (75\%) + AL (25\%); $\mathrm{S}_{3}$ - TS (75\%) + AL (15\%) + $\mathrm{EB}(10 \%) ; \mathrm{S}_{4}-\mathrm{TS}(60 \%)+\mathrm{AL}(20 \%)+\mathrm{EB}(20 \%) ; \mathrm{S}_{5}-\mathrm{TS}(52,5 \%)+\mathrm{AL}(17,5 \%)+\mathrm{EB}(30 \%) ; \mathrm{S}_{6}-\mathrm{TS}(45 \%)+$ $\mathrm{AL}(15 \%)+\mathrm{EB}(40 \%) ; \mathrm{S} 7-\mathrm{TS}(37,5 \%)+\mathrm{AL}(12,5 \%)+\mathrm{EB}(50 \%) . \mathrm{TS}=$ terra de subsolo; $\mathrm{AL}=$ areia lavada; $\mathrm{EB}=$ esterco bovino. 
TABELA 3: Altura de mudas de Spondias tuberosa em diferentes substratos e volumes de recipientes, 78 dias após a repicagem.

TABLE 3: Seedling height of Spondias tuberosa in different substrates and container volumes, 78 days after the transplant.

\begin{tabular}{lll}
\hline \multicolumn{1}{c}{ Substrato } & \multicolumn{2}{c}{ Altura $\left(\mathrm{cm} \mathrm{planta}^{-1}\right)$} \\
\cline { 2 - 3 } & $1900 \mathrm{~cm}^{3}$ & $5000 \mathrm{~cm}^{3}$ \\
\hline $\mathrm{S}_{1}-\mathrm{TS}(100 \%)$ & $15,61 \mathrm{Bd}$ & $19,65 \mathrm{Ad}$ \\
$\mathrm{S}_{2}-\mathrm{TS}(75 \%)+\mathrm{AL}(25 \%)$ & $13,79 \mathrm{Bd}$ & $17,66 \mathrm{Ad}$ \\
$\mathrm{S}_{3}-\mathrm{TS}(75 \%)+\mathrm{AL}(15 \%)+\mathrm{EB}(10 \%)$ & $27,01 \mathrm{Bc}$ & $34,45 \mathrm{Ac}$ \\
$\mathrm{S}_{4}-\mathrm{TS}(60 \%)+\mathrm{AL}(20 \%)+\mathrm{EB}(20 \%)$ & $30,12 \mathrm{Bbc}$ & $40,24 \mathrm{Aab}$ \\
$\mathrm{S}_{5}-\mathrm{TS}(52,5 \%)+\mathrm{AL}(17,5 \%)+\mathrm{EB}(30 \%)$ & $35,66 \mathrm{Aa}$ & $37,00 \mathrm{Abc}$ \\
$\mathrm{S}_{6}-\mathrm{TS}(45 \%)+\mathrm{AL}(15 \%)+\mathrm{EB}(40 \%)$ & $35,95 \mathrm{Ba}$ & $44,52 \mathrm{Aa}$ \\
$\mathrm{S}_{7}-\mathrm{TS}(37,5 \%)+\mathrm{AL}(12,5 \%)+\mathrm{EB}(50 \%)$ & $35,20 \mathrm{Aab}$ & $40,22 \mathrm{Aab}$ \\
\hline
\end{tabular}

Em que: letras maiúsculas comparam recipientes (colunas) dentro de cada substrato (linhas) e letras minúsculas comparam substratos (linhas) em cada volume de recipiente (colunas). Médias seguidas da mesma letra não diferem estatisticamente entre si pelo teste de Tukey a 5\% de probabilidade. Em que: $\mathrm{TS}=$ terra de subsolo; $\mathrm{AL}=$ areia lavada; $\mathrm{EB}=$ esterco bovino.

incremento na altura de $5,79 \mathrm{~cm}$ em comparação com as que cresceram nos recipientes com 1900 $\mathrm{cm}^{3}$. As maiores alturas verificadas no recipiente de $5000 \mathrm{~cm}^{3}$ podem estar relacionadas ao fato deste proporcionar maior espaço para deposição de substrato e condições para as plantas terem maior crescimento e desenvolvimento das raízes refletindo, por fim, em um aumento na altura. Plantas de Spondias tuberosa provenientes do substrato contendo apenas terra de subsolo ou a combinação desta com a areia lavada não obtiveram condições favoráveis para um crescimento de altura. Isso pode estar relacionado com os baixos teores de fósforo presentes nesses substratos. Marenco e Lopes (2007) destacaram que depois do nitrogênio, o fósforo é o elemento que mais limita o crescimento das plantas.

Carvalho Filho et al. (2004) enfatizaram que a terra de subsolo tem sido comumente utilizada como principal material no preenchimento de embalagens plásticas, entretanto, geralmente apresenta baixos níveis de nutrientes. Os principais efeitos dos substratos se manifestam nas raízes proporcionando influência sobre a altura das plantas (HARTMANN et al., 1990). Negreiros et al. (2004), ao avaliarem a influência de substratos na formação de porta enxertos de gravioleira (Annona muricata L.), constataram que os substratos que continham esterco em sua composição proporcionaram maiores valores para altura de plantas, ressaltando que o esterco fornece melhores condições nutricionais para o crescimento das mudas. Braga Júnior et al. (2010), ao avaliarem a influência de diferentes substratos sobre a emergência de plântulas de juazeiro (Zizyphus joazeiro Mart.), constataram que a utilização de esterco bovino e terra vegetal na proporção de 1:1 proporcionou um dos maiores valores para o comprimento da parte aérea da referida espécie.

Tanto para o diâmetro do colo (DC) quanto para a relação altura/diâmetro do colo (H/DC) não houve interação entre os recipientes e substratos avaliados. Por sua vez, os substratos iinfluenciaram significativamente nas referidas características, cujo teste de média está presente na Tabela 4.

O diâmetro do colo é uma das características cuja análise permite indicar a capacidade de uma muda sobreviver no campo e deve ser utilizado como um dos melhores indicadores de padrão de qualidade (STURION, 1981; DANIEL et al., 1997). Os substratos com esterco bovino curtido proporcionaram os maiores valores de diâmetro do colo de mudas de Spondias tuberosa independentemente do recipiente utilizado. Dentre os substratos avaliados, o maior valor para o diâmetro do colo foi observado em mudas de umbuzeiro que se desenvolveram no substrato $\mathrm{S}_{6}$ - Terra de subsolo (45\%) + Areia lavada (15\%) + Esterco bovino (40\%), cujo valor correspondeu a $8,18 \mathrm{~mm}$ planta $^{-1}$.

A formulação de substratos com componentes de fácil aquisição como a terra e a areia, bem como a utilização de material orgânico (esterco curtido de curral) que confere características benéficas na composição do substrato, pode tanto 
TABELA 4: Diâmetro do colo (DC) e relação altura/diâmetro do colo (H/DC) de mudas de Spondias tuberosa em diferentes substratos, 78 dias após a repicagem.

TABLE 4: Collar diameter (DC) and relation height/collar diameter (H/DC) of Spondias tuberosa seedlings in different subtracts, 78 days after the transplant.

\begin{tabular}{lcc}
\hline \multicolumn{1}{c}{ Substrato } & DC $\left(\mathrm{mm} \mathrm{planta}^{-1}\right)$ & $\mathrm{H} / \mathrm{DC}$ \\
\hline $\mathrm{S}_{1}-\mathrm{TS}(100 \%)$ & $4,50 \mathrm{~b}$ & $3,88 \mathrm{~b}$ \\
$\mathrm{~S}_{2}-\mathrm{TS}(75 \%)+\mathrm{AL}(25 \%)$ & $4,31 \mathrm{~b}$ & $3,77 \mathrm{~b}$ \\
$\mathrm{~S}_{3}-\mathrm{TS}(75 \%)+\mathrm{AL}(15 \%)+\mathrm{EB}(10 \%)$ & $6,77 \mathrm{a}$ & $4,53 \mathrm{ab}$ \\
$\mathrm{S}_{4}-\mathrm{TS}(60 \%)+\mathrm{AL}(20 \%)+\mathrm{EB}(20 \%)$ & $7,82 \mathrm{a}$ & $4,50 \mathrm{ab}$ \\
$\mathrm{S}_{5}-\mathrm{TS}(52,5 \%)+\mathrm{AL}(17,5 \%)+\mathrm{EB}(30 \%)$ & $7,59 \mathrm{a}$ & $4,56 \mathrm{ab}$ \\
$\mathrm{S}_{6}-\mathrm{TS}(45 \%)+\mathrm{AL}(15 \%)+\mathrm{EB}(40 \%)$ & $8,18 \mathrm{a}$ & $5,20 \mathrm{a}$ \\
$\mathrm{S}_{7}-\mathrm{TS}(37,5 \%)+\mathrm{AL}(12,5 \%)+\mathrm{EB}(50 \%)$ & $7,61 \mathrm{a}$ & $4,96 \mathrm{ab}$ \\
\hline
\end{tabular}

Em que: médias seguidas da mesma letra não diferem estatisticamente entre si pelo teste de Tukey a 5\% de probabilidade. $\mathrm{Em}$ que: $\mathrm{TS}=$ terra de subsolo; $\mathrm{AL}=$ areia lavada; $\mathrm{EB}=$ esterco bovino.

promover a redução do custo final como favorecer a produção de mudas de qualidade (PIO et al., 2004; BARDIVIESSO et al., 2011). Os menores valores do diâmetro do colo foram registrados nas mudas que cresceram nos substratos $\mathrm{S}_{1}$ - Terra de subsolo $100 \%$ e $\mathrm{S}_{2}$ - Terra $(75 \%)+$ Areia lavada $(25 \%)$, os quais corresponderam a 4,50 e $4,31 \mathrm{~mm}$ planta-1; respectivamente.

Embora a terra de subsolo seja um dos substratos mais utilizados para a produção de mudas em viveiro, a mesma apresenta baixa disponibilidade de nutrientes e, muitas vezes, acidez elevada, proporcionando mudas com características indesejáveis (TUCCI et al., 2009). Todos os substratos utilizados, com exceção do substrato $\mathrm{S}_{6}$, apresentaram $\mathrm{pH}$ entre 4,4 e 5,0. Embora a acidez seja uma das características mais limitantes no que tange a disponibilização de nutrientes para as plantas, Kämpf (2000) destacou que há maior disponibilidade de nutrientes em substratos de base orgânica quando os mesmos apresentam pH (em água) situado entre 5,2 e 5,5, o que possivelmente explica os menores valores para algumas variáveis avaliadas em plantas que cresceram no substrato $\mathrm{S}_{7}$, já que este teve um aumento excessivo do $\mathrm{pH}$. Apesar da acidez dos substratos, os bons resultados para o crescimento de mudas de Spondias tuberosa naqueles contendo esterco bovino, está relacionado tanto pela maior fertilidade conferida pelo material orgânico, quanto ao fato de que a espécie é nativa da caatinga, bioma caracterizado por apresentar solos, com raras exceções, rasos, pobres em nutrientes e de acidez elevada (ALVES et al., 2009).

Quanto ao recipiente utilizado, o de capacidade de $5000 \mathrm{~cm}^{3}$ conferiu maior diâmetro do colo independentemente do substrato avaliado. Para a relação altura/diâmetro do colo, Carneiro (1995) recomendou que os valores dessa relação situem-se entre 5,4 e 8,1 para a espécie Pinus taeda, indicando mudas com crescimento com maior equilíbrio e ideais para o plantio. Independentemente dos volumes de recipientes avaliados, dentre os substratos testados, o que proporcionou uma relação H/DC mais próxima da proposta pelo citado autor foi o substrato $\mathrm{S}_{6}$ - Terra de subsolo (45\%) + Areia lavada (15\%) + Esterco bovino (40\%).

Quanto ao diâmetro médio de xilopódios (Tabela 5), o maior valor foi registrado em plantas de umbuzeiro (Spondias tuberosa) que se desenvolveram no recipiente de $1900 \mathrm{~cm}^{3}$ e no substrato $\mathrm{S}_{5}-$ Terra $(52,5 \%)+$ Areia $(17,5 \%)+$ Esterco bovino (30\%). Houve diferença estatística para os recipientes que continham substratos com esterco, com exceção do substrato $\mathrm{S}_{6}$ - Terra (45\%) + Areia $(15 \%)+$ Esterco bovino (40\%). Silva et al. (2005) destacaram que, durante a fase de crescimento e formação do xilopódio, há uma maior exigência de nutrientes para proporcionar melhor condição para a planta resistir ao período seco. Independentemente do substrato, plantas de Spondias tuberosa, que se desenvolveram nos recipientes de $5000 \mathrm{~cm}^{3}$, obtiveram apenas um incremento de $0,16 \mathrm{~cm}$ no diâmetro médio de xilopódios quando comparadas com aquelas dos recipientes de $1900 \mathrm{~cm}^{3}$.

A incorporação de $25 \%$ de areia lavada à terra de subsolo resultou no menor valor médio de diâmetro de xilopódio para plantas de Spondias tuberosa que se desenvolveram no recipiente de 
TABELA 5: Diâmetro de xilopódios de mudas de Spondias tuberosa em diferentes substratos e volumes de recipientes, 78 dias após a repicagem.

TABLE 5: Xylopodium diameter of Spondias tuberosa seedlings in different substrates and volumes of containers, 78 days after the transplant.

\begin{tabular}{lcc}
\hline \multicolumn{1}{c}{ Substrato } & \multicolumn{2}{c}{ Diâmetro de xilopódios $\left(\mathrm{cm} \mathrm{planta}^{-1}\right)$} \\
\cline { 2 - 3 } & $1900 \mathrm{~cm}^{3}$ & $5000 \mathrm{~cm}^{3}$ \\
\hline $\mathrm{S}_{1}-\mathrm{TS}(100 \%)$ & $2,05 \mathrm{Ade}$ & $2,09 \mathrm{Ab}$ \\
$\mathrm{S}_{2}-\mathrm{TS}(75 \%)+\mathrm{AL}(25 \%)$ & $1,87 \mathrm{Ae}$ & $2,11 \mathrm{Ab}$ \\
$\mathrm{S}_{3}-\mathrm{TS}(75 \%)+\mathrm{AL}(15 \%)+\mathrm{EB}(10 \%)$ & $2,35 \mathrm{Bcd}$ & $2,85 \mathrm{Aa}$ \\
$\mathrm{S}_{4}-\mathrm{TS}(60 \%)+\mathrm{AL}(20 \%)+\mathrm{EB}(20 \%)$ & $2,79 \mathrm{Bbc}$ & $3,17 \mathrm{Aa}$ \\
$\mathrm{S}_{5}-\mathrm{TS}(52,5 \%)+\mathrm{AL}(17,5 \%)+\mathrm{EB}(30 \%)$ & $3,31 \mathrm{Aa}$ & $2,90 \mathrm{Ba}$ \\
$\mathrm{S}_{6}-\mathrm{TS}(45 \%)+\mathrm{AL}(15 \%)+\mathrm{EB}(40 \%)$ & $3,06 \mathrm{Aab}$ & $3,01 \mathrm{Aa}$ \\
$\mathrm{S}_{7}-\mathrm{TS}(37,5 \%)+\mathrm{AL}(12,5 \%)+\mathrm{EB}(50 \%)$ & $2,53 \mathrm{Bc}$ & $3,01 \mathrm{Aa}$ \\
\hline
\end{tabular}

Em que: letras maiúsculas comparam recipientes (colunas) dentro de cada substrato (linhas) e letras minúsculas comparam substratos (linhas) em cada tamanho de recipiente (colunas). Médias seguidas da mesma letra não diferem estatisticamente entre si pelo teste de Tukey a $5 \%$ de probabilidade. Em que: $\mathrm{TS}=$ terra de subsolo; $\mathrm{AL}=$ areia lavada; $\mathrm{EB}=$ esterco bovino.

$1900 \mathrm{~cm}^{3}$, cujo valor correspondeu a $1,87 \mathrm{~cm}$ planta $^{-1}$. Os recipientes de maior volume geralmente são utilizados para espécies que apresentam crescimento lento ou que permanecerão mais tempo no viveiro. Além disso, seu uso é intensificado quando há necessidade de obtenção de mudas com maior desenvolvimento, sendo necessário, muitas vezes, associar o recipiente a um substrato que proporcione a obtenção dos resultados esperados (CUNHA et al., 2005).

O crescimento em diâmetro do xilopódio é um indicativo de que as mudas produzidas apresentaram uma boa reserva de água e sais minerais, características indispensáveis para que a planta tolere os estresses abióticos resultantes da etapa de plantio no local definitivo, o que é muito importante, principalmente quando o objetivo da produção de mudas é a recuperação de áreas desmatadas da caatinga. Quanto ao comprimento de raiz (CR) de plantas de umbuzeiro, os substratos $\mathrm{S}_{4}$ - Terra de subsolo $(60 \%)+$ Areia lavada $(20 \%)+$ Esterco bovino (20\%) e $\mathrm{S}_{5}$ - Terra $(52,5 \%)+$ Areia $(17,5 \%)+$ Esterco bovino (30\%), promoveram os maiores valores, diferindo estatisticamente, apenas do substrato $\mathrm{S}_{7}$ - Terra de subsolo $(37,5 \%)$ + Areia lavada (12,5\%) + Esterco bovino (50\%) (Tabela 6). Plantas que se desenvolveram em substratos contendo esterco tiveram um incremento de $2,68 \mathrm{~cm}$ em comparação com as que não $o$ tinham em sua composição. $O$ esterco auxilia no fornecimento de nutrientes para as plantas resultando maiores ganhos de comprimento de raiz.

TABELA 6: Comprimento de raiz de mudas de Spondias tuberosa em diferentes substratos, 78 dias após a repicagem.

TABLE 6: Root length of Spondias tuberosa seedlings in different substrates, 78 days after the transplant.

\begin{tabular}{lc}
\hline \multicolumn{1}{c}{ Substrato } & Comprimento da raiz $\left(\mathrm{cm} \mathrm{planta}^{-1}\right)$ \\
\hline $\mathrm{S}_{1}-\mathrm{TS}(100 \%)$ & $27,79 \mathrm{ab}$ \\
$\mathrm{S}_{2}-\mathrm{TS}(75 \%)+\mathrm{AL}(25 \%)$ & $27,20 \mathrm{ab}$ \\
$\mathrm{S}_{3}-\mathrm{TS}(75 \%)+\mathrm{AL}(15 \%)+\mathrm{EB}(10 \%)$ & $31,24 \mathrm{ab}$ \\
$\mathrm{S}_{4}-\mathrm{TS}(60 \%)+\mathrm{AL}(20 \%)+\mathrm{EB}(20 \%)$ & $31,77 \mathrm{a}$ \\
$\mathrm{S}_{5}-\mathrm{TS}(52,5 \%)+\mathrm{AL}(17,5 \%)+\mathrm{EB}(30 \%)$ & $32,47 \mathrm{a}$ \\
$\mathrm{S}_{6}-\mathrm{TS}(45 \%)+\mathrm{AL}(15 \%)+\mathrm{EB}(40 \%)$ & $30,08 \mathrm{ab}$ \\
$\mathrm{S}_{7}-\mathrm{TS}(37,5 \%)+\mathrm{AL}(12,5 \%)+\mathrm{EB}(50 \%)$ & $25,36 \mathrm{~b}$
\end{tabular}

Em que: médias seguidas da mesma letra não diferem estatisticamente entre si pelo teste de Tukey a $5 \%$ de probabilidade. $\mathrm{TS}=$ terra de subsolo; $\mathrm{AL}=$ areia lavada; $\mathrm{EB}=$ esterco bovino. 
Tucci et al. (2009) destacaram que um substrato com baixa fertilidade interfere na qualidade de mudas no viveiro e aumenta o índice de mortalidade das mesmas quanto colocadas no local definitivo.

Com relação à massa seca da parte aérea de plantas de Spondias tuberosa, o recipiente com volume de $5000 \mathrm{~cm}^{3}$ proporcionou os maiores valores em relação ao recipiente de $1900 \mathrm{~cm}^{3}$, sobretudo para os substratos contendo esterco em sua composição (Tabela 7), os quais apresentaram maiores teores de fósforo e cálcio. Silva et al. (2005), ao avaliarem as limitações nutricionais do crescimento de mudas de Spondias tuberosa em Latossolo Vermelho distrófico, constataram que a omissão de nitrogênio e fósforo resultou na menor produção de massa seca da parte aérea, seguida da falta de cálcio. Para os substratos $\mathrm{S}_{1}$ - Terra de subsolo (100\%) e $\mathrm{S}_{2}$ - Terra de subsolo $(75 \%)+$ Areia $(25 \%)$ não houve diferença estatística para os dois tipos de recipientes utilizados, sendo que os mesmos proporcionaram os menores valores de massa seca da parte aérea. Isso ocorreu devido a esses substratos não terem em sua constituição material orgânico, o qual proporcionaria maior concessão de nutrientes à planta resultando em maiores e satisfatórios ganhos de biomassa da parte aérea. A utilização de material orgânico na produção de mudas de espécies florestais tem proporcionado satisfatórios resultados. Cunha et al. (2006) comparando substratos com a mesma proporção de material orgânico na produção de mudas de Acacia sp. observaram mudas com maior qualidade quando foi utilizado esterco bovino.

Os adubos orgânicos, a exemplo do esterco bovino curtido, são fontes de nutrientes frequentemente incorporados a substratos, atuando significativamente na melhoria dos atributos físicos e químicos (ARTUR et al., 2007). Quanto à massa seca da parte aérea, plantas de Spondias tuberosa que cresceram em recipiente de $5000 \mathrm{~cm}^{3}$, a incorporação de $30 \%, 40 \%$ e $50 \%$ de esterco bovino à terra de subsolo mais areia lavada proporcionou os maiores valores médios, os quais não diferiram estatisticamente dentro do referido recipiente. Os valores corresponderam a 10,$41 ; 11,10$ e $10,78 \mathrm{~g}$ planta $^{-1}$ nos substratos $\mathrm{S}_{5}, \mathrm{~S}_{6}$ e $\mathrm{S}_{7}$, respectivamente. Silva et al. (2009), ao avaliarem diferentes substratos na produção de mudas de mangabeira (Hancornia speciosa Gomes), constataram que a proporção de $20 \%$ e $40 \%$ de esterco aos substratos Plantimax ${ }^{\circledR}$ e solo, respectivamente, promoveu os maiores valores de massa seca de parte aérea.

Quanto à massa seca de raízes de plantas de umbuzeiro (Spondias tuberosa), os maiores valores foram provenientes de mudas que se desenvolveram no substrato $\mathrm{S}_{4}$ - Terra de subsolo $(60 \%)+$ Areia lavada $(20 \%)+$ Esterco bovino (20\%) e em recipiente de $5000 \mathrm{~cm}^{3}$. No que diz respeito aos recipientes com volume de $1900 \mathrm{~cm}^{3}$, a incorporação de $30 \%$ de esterco bovino ao substrato contendo $52,5 \%$ de terra e $17,5 \%$ de areia $\left(\mathrm{S}_{5}\right)$ resultou em um maior valor, correspondendo a 6,72 $\mathrm{g}_{\text {planta }}{ }^{-1}$. Para esse mesmo substrato, houve um maior equilíbrio em relação aos dois recipientes avaliados. Resultados

TABELA 7: Massa seca da parte aérea e de raízes de mudas de Spondias tuberosa em diferentes substratos e volumes de recipientes 78 dias após a repicagem.

TABLE 7: Dry mass of shoots and roots of Spondias tuberosa seedlings in different substrates and volumes of containers 78 days after the transplant.

\begin{tabular}{lcclc}
\hline \multirow{2}{*}{ Substrato } & \multicolumn{2}{c}{ MSPA $\left(\mathrm{g} \mathrm{planta} \mathrm{p}^{-1}\right)$} & \multicolumn{2}{c}{ MSRA $\left(\mathrm{g} \mathrm{planta}^{-1}\right)$} \\
\cline { 2 - 5 } & $1900 \mathrm{~cm}^{3}$ & $5000 \mathrm{~cm}^{3}$ & $1900 \mathrm{~cm}^{3}$ & $5000 \mathrm{~cm}^{3}$ \\
\hline $\mathrm{S}_{1}-\mathrm{TS}(100 \%)$ & $0,90 \mathrm{Ad}$ & $1,25 \mathrm{Ac}$ & $1,57 \mathrm{Ac}$ & $1,63 \mathrm{Ac}$ \\
$\mathrm{S}_{2}-\mathrm{TS}(75 \%)+\mathrm{AL}(25 \%)$ & $0,66 \mathrm{Ad}$ & $0,96 \mathrm{Ac}$ & $1,17 \mathrm{Ac}$ & $1,56 \mathrm{Ac}$ \\
$\mathrm{S}_{3}-\mathrm{TS}(75 \%)+\mathrm{AL}(15 \%)+\mathrm{EB}(10 \%)$ & $2,65 \mathrm{Bcd}$ & $4,76 \mathrm{Ab}$ & $3,35 \mathrm{Abc}$ & $4,51 \mathrm{Ab}$ \\
$\mathrm{S}_{4}-\mathrm{TS}(60 \%)+\mathrm{AL}(20 \%)+\mathrm{EB}(20 \%)$ & $3,75 \mathrm{Bbc}$ & $5,80 \mathrm{Ab}$ & $4,26 \mathrm{Bb}$ & $7,21 \mathrm{Aa}$ \\
$\mathrm{S}_{5}-\mathrm{TS}(52,5 \%)+\mathrm{AL}(17,5 \%)+\mathrm{EB}(30 \%)$ & $6,04 \mathrm{Ba}$ & $10,41 \mathrm{Aa}$ & $6,72 \mathrm{Aa}$ & $6,26 \mathrm{Aab}$ \\
$\mathrm{S}_{6}-\mathrm{TS}(45 \%)+\mathrm{AL}(15 \%)+\mathrm{EB}(40 \%)$ & $6,56 \mathrm{Ba}$ & $11,10 \mathrm{Aa}$ & $5,44 \mathrm{Aab}$ & $5,35 \mathrm{Aab}$ \\
$\mathrm{S}_{7}-\mathrm{TS}(37,5 \%)+\mathrm{AL}(12,5 \%)+\mathrm{EB}(50 \%)$ & $5,89 \mathrm{Bab}$ & $10,78 \mathrm{Aa}$ & $4,64 \mathrm{Aab}$ & $4,64 \mathrm{Ab}$ \\
\hline
\end{tabular}

Em que: letras maiúsculas comparam recipientes (colunas) dentro de cada substrato (linhas) e letras minúsculas comparam substratos (linhas) em cada tamanho de recipiente (colunas). Médias seguidas da mesma letra não diferem estatisticamente entre si pelo teste de Tukey a $5 \%$ de probabilidade. Em que: TS = terra de subsolo; $\mathrm{AL}=$ areia lavada; $\mathrm{EB}=$ esterco bovino; $\mathrm{MSPA}=$ massa seca da parte aérea; $\mathrm{MSR}=$ massa seca da raiz. 
satisfatórios obtidos com o uso do esterco estão relacionados com o suprimento de nutrientes feito pela presença da matéria orgânica, bem como nas melhorias da capacidade de retenção de água do mesmo (SILVA JÚNIOR e GIORGI, 1992; SILVA et al., 2010). As características químicas do substrato podem influenciar no ganho de biomassa das raízes e consequentemente na massa seca de plantas de espécies vegetais.

Embora o comprimento de raiz tenha sido superior a $20 \mathrm{~cm}$ em todos os substratos avaliados e independentemente dos recipientes, a massa seca de raízes de mudas de Spondias tuberosa foi bastante inferior nos substratos $\mathrm{S}_{1}$ - Terra de subsolo $(100 \%)$ e $\mathrm{S}_{2}$ - Terra de subsolo (75\%) + Areia lavada (25\%) quando comparados aos demais substratos, sem levar em consideração o recipiente utilizado. Isso se explica através do fato de que, com exceção dos referidos substratos, os demais proporcionaram a formação de uma maior quantidade de biomassa radicular pela maior ocorrência de raízes secundárias. Souza et al. (2005) enfatizaram que os substratos enriquecidos com material orgânico, por fornecerem maior quantidade de nutrientes, proporcionam um melhor desenvolvimento de raízes, além de apresentarem uma maior capacidade de retenção de água. Dessa forma, ocorre uma maior translocação de assimilados para as raízes resultando em um maior crescimento.

$\mathrm{O}$ substrato $\mathrm{S}_{4}$ - Terra de subsolo $(60 \%)+$ Areialavada (20\%)+Estercobovino(20\%) foioúnico que conferiu diferença estatisticamente significativa sobre o desenvolvimento e consequentemente na formação de biomassa radicular em relação aos dois recipientes avaliados. No recipiente de $5000 \mathrm{~cm}^{3}$, o referido substrato proporcionou valor médio de 7,21 g planta $^{-1}$ contra 4,26 g planta $^{-1}$ no recipiente de $1900 \mathrm{~cm}^{3}$. Esse resultado mostra que, em condições bastante específicas de escolha de recipientes, um mesmo substrato pode proporcionar produção de biomassa radicular bastante expressiva.

\section{CONCLUSÃO}

Independentemente dos volumes de recipientes avaliados, o substrato Terra de subsolo $(45 \%)+$ Areia lavada $(15 \%)+$ Esterco bovino (40\%) proporciona a melhor relação altura/diâmetro do colo aos 78 dias após a repicagem de plântulas de Spondias tuberosa, sendo recomendado para a produção de mudas da referida espécie.

O recipiente de $1900 \mathrm{~cm}^{3}$ é indicado para produção de mudas de Spondias tuberosa por requerer menor quantidade de substrato.

\section{REFERÊNCIAS BIBLIOGRÁFICAS}

ALBUQUERQUE, M. C. F. et al. Influência da temperatura e do substrato na germinação de sementes de saguaraji (Colubrina glandulosa Perk. - Rhamnaceae). Revista Brasileira de Sementes, Brasília, v. 20, n. 2, p. 108-111, 1998.

ALVES, J. J. A.; ARAÚJO, M. A.; NASCIMENTO, S. S. Degradação da caatinga: uma investigação ecogeográfica. Revista Caatinga, Mossoró, v. 22, n. 3, p. 126-135, 2009.

ARTUR, A. G. et al. Esterco bovino e calagem para formação de mudas de guanandi. Pesquisa Agropecuária Brasileira, Brasília, v. 42, n. 6, p. 843-850, 2007.

BARDIVIESSO, D. M. et al. Diferentes substratos e recipientes na produção de mudas de guabiroba (Campomanesia pubescens O. Berg). Revista Científica Eletrônica de Agronomia, Garça, v. 18, n. 1, p. 52-59, 2011.

BRAGA JÚNIOR, J. M.; BRUNO, R. L.A.; ALVES, E. U. Emergência de plântulas de Zizyphus joazeiro Mart (Rhamnaceae) em função de substratos. Revista Árvore, Viçosa, v. 34, n. 4, p. 609-616, 2010.

CARNEIRO, J. G. A. Produção e controle de qualidade de mudas florestais. Curitiba: UFPR/ FUPEF, 1995. $451 \mathrm{p}$.

CARVAlHO FILHO, J. L. S. et al. Produção de mudas de jatobá (Hymenaea courbaril L.) em diferentes ambientes e composições de substratos. Cerne, Lavras, v. 9, n. 1, p. 109-118, 2003.

CARVALHO FILHO, J. L. S.; ARRIGONIBLANK, M. F.; BLANK, A. F. Produção de mudas de angelim (Andira fraxinifolia Benth.) em diferentes ambientes, recipientes e composições de substratos. Revista Ciência Agronômica, Fortaleza, v. 35, n. 1, p. 61-67, 2004.

CAVALCANTI, N. B.; RESENDE, G. M.; BRITO, L. T. L. Emergência e crescimento do imbuzeiro (Spondias tuberosa) em diferentes substratos. Revista Ceres, Viçosa, v. 49, n. 282, p. 97-108, 2002a.

CAVALCANTI, N. B.; RESENDE, G. M.; BRITO, L. T. L. Levantamento da produção de xilopódio e os efeitos de sua retirada sobre a frutificação e persistência de plantas nativas de imbuzeiro (Spondias tuberosa Arr. Cam.). Ciência e Agrotecnologia, Lavras. v. 26, n. 5, p.927-942, 
$2002 b$.

CAVALCANTI, N. B.; RESENDE, G. M.; BRITO, L. T. L. Processamento do fruto do imbuzeiro (Spondias tuberosa Arr. Cam.). Ciência e Agrotecnologia, Lavras, v. 24, n. 1, p.252-259, 2000.

COSTA, N. P. et al. Efeito do estádio de maturação do fruto e do tempo de pré-embebição de endocarpos na germinação de sementes de umbuzeiro (Spondias tuberosa Arr. Câm.). Revista Brasileira de Fruticultura, Jaboticabal, v. 23, n. 3, p. 738-741, 2001.

CUNHA, A. M. et al. Efeito de diferentes substratos sobre o desenvolvimento de mudas de Acacia sp. Revista Árvore, Viçosa, v. 30, n. 2, p. 207-214, 2006.

CUNHA, A. O. et al. Efeitos de substratos e das dimensões dos recipientes na qualidade das mudas de Tabebuia impetiginosa (Mart. Ex D.C.) Standl. Revista Árvore, Viçosa, v. 29, n. 4, p. 507-516, 2005.

DANIEL, O. et al. Aplicação de fósforo em mudas de Acacia mangium Willd. Revista Árvore, Viçosa v. 21, p. 163-168, 1997.

FERRAZ, M. V.; CENTURION, J. F.; BEUTLER, A. N. Caracterização física e química de alguns substratos comerciais. Acta Scientiarum Agronomy, Maringá, v. 27, n. 2, p. 209-214, 2005. FERREIRA, D.F. Sisvar: Versão 5.1 (Build 72). DEX/UFLA. 2007.

FOLEGATTI, M. I. S. et al. Aproveitamento industrial do umbu: processamento de geléia e compota. Ciência e Agrotecnologia, Lavras. v. 27, n. 6, p. 1308-1314, 2003.

HARTMANN, H. T.; KESTER, D. E.; DAVIES JÚNIOR, F. T. Plant propagation: principles and practices. 5. ed. New York: EnglewoodClipps/ Prentice Hall, 1990. 647p.

JOSÉ, A. C.; DAVIDE, A. C.; OLIVEIRA, S. L. Produção de mudas de aroeira (Schinus terebinthifolius Raddi) para recuperação de áreas degradadas pela mineração de bauxita. Cerne, Lavras, v. 11, n. 2, p. 187-196, 2005.

KÄMPF, A. N. Seleção de materiais para uso como substrato. In: KÄMPF NA; FERMINO MH. (Eds.)

Substratos para plantas: a base da produção vegetal em recipientes. Porto Alegre: Gênesis, 2000. p.139-145.

LIMA, R. L. S. et al. Substratos para produção de mudas de mamoneira compostos por misturas de cinco fontes de matéria orgânica. Ciência e agrotecnologia, Lavras, v. 30, n. 3, p. 474-479,
$2006 a$.

LIMA, R. L. S. et al. Volume de recipientes e composição de substratos para produção de mudas de mamoneira. Ciência e Agrotecnologia, Lavras, v. 30, n. 3, p. 480-486, 2006 b.

LORENZI, H. Árvores brasileiras: manual de identificação e cultivo de plantas arbóreas nativas do Brasil. 2 ${ }^{\mathrm{a}}$ edição. Nova Odessa, SP: Editora Plantarum, 1998. p. 10.

MARENCO, R. A.; LOPES, N. F. 2007. Fisiologia vegetal: fotossíntese, respiração, relações hídricas e nutrição mineral. 2 ed. Editora UFV, Viçosa, MG. 363 pp.

MELO, A. S. et al. Desenvolvimento de portaenxertos de umbuzeiro em resposta à adubação com nitrogênio e fósforo. Ciência Rural, Santa Maria, v. 35, n. 2, p. 324-331, 2005.

NEGREIROS, J. R. S. et al. Influência de substratos na formação de porta-enxerto de gravioleira (Annona muricata L.). Ciência e Agrotecnologia, Lavras, v. 28, n. 3, p. 530-536, 2004.

NICOLOSO, F. T. et al. Recipientes e substratos na produção de mudas de Maytenus ilicifolia e Apuleia leiocarpa. Ciência Rural, Santa Maria, v. 30, n. 6, p. 987-992, 2000.

PAULA, L. A. et al. Efeito do ácido indolbutírico e raizon no enraizamento de estacas herbáceas e lenhosas de umbuzeiro. Acta Sciencia Agronômica. Maringá, v. 29, n. 3, p. 411-414, 2007.

PEREIRA, P. C. et al. Tamanho de recipientes e tipos de substrato na qualidade de mudas de tamarindeiro. Revista Verde, Mossoró, v. 5, n. 3, p. 136-142, 2010.

PIO, R. et al. Efeito de diferentes substratos no crescimento de mudas de nespereira. Revista Brasileira de Agrociência, Pelotas, v. 10, n. 3, p. 309-312, 2004.

RODRIGUES, A. C. C. et al. Efeito do substrato e luminosidade na germinação de Anadenanthera columbrina (Fabaceae, Mimosoideae). Revista Árvore, Viçosa, v. 31, n. 2, p. 187-193, 2007.

SANTANA, J. A. S.; SOUTO, J. S. Diversidade e estrutura fitossociológica da caatinga na Estação Ecológica do Seridó - RN. Revista de Biologia e Ciências da Terra, Campina Grande, v. 6, n. 2, p. 232-242, 2006.

SILVA JÚNIOR, A. A.; GIORGI, E. Substrato alternativo para a produção de mudas de tomate. Florianópolis: EPAGRI, 1992. 23p. (Boletim Técnico, 1992).

SILVA, E. A. et al. Composição de substratos e tamanho de recipientes na produção e qualidade 
das mudas de maracujazeiro 'amarelo'. Ciência e Agrotecnologia, Lavras, v. 34, n. 3, p. 588-595, 2010.

SILVA, E. A. et al. Efeito de diferentes substratos na produção de mudas de mangabeira (Hancornia speciosa). Revista Brasileira de Fruticultura, Jaboticabal, v. 31, n. 3, p. 925-929, 2009.

SILVA, E. B., GONÇALVES, N. P.; PINHO, P. J. Limitações nutricionais para crescimento de mudas de umbuzeiro em Latossolo Vermelho distrófico no Norte de Minas. Acta Scientiarum. Agronomy, Maringá, v. 27, n. 1, p. 55-59, 2005.

SMIDERLE, O. J. et al. Produção de mudas de alface, pepino e pimentão em substratos combinando areia, solo e Plantmax ${ }^{\circledR}$. Horticultura Brasileira, Vitória da Conquista, v. 19, n. 3, p. 253-257, 2001. SOUZA, V. C. et al. Produção de Mudas de Ipê- amarelo (Tabebuia serratifolia (Vahl.) Nich.) em diferentes substratos e tamanhos de recipientes. Agropecuária Técnica, Areia, v. 26, n. 2, p. 98-108, 2005.

STURION, J. A. Influência do recipiente e do método de semeadura na formação de mudas de Mimosa scabrella Bentham. Boletim de Pesquisa Florestal, Colombo, n. 2, p. 69-88, 1981.

TUCCI, C. A. F.; LIMA, H. N.; LESSA, J. F. Adubação nitrogenada na produção de mudas de mogno (Swietenia macrophylla King). Acta Amazonica, Manaus, v. 39, n. 2, p. 289-294, 2009. XAVIER, K. R F. et al. Impactos pós-fogo na regeneração natural em um fragmento de floresta ombrófila aberta no município de Areia, Paraíba, Brasil. Revista brasileira de Biociências, Porto Alegre, v. 9, n. 3, p. 257-264, 2011. 